\title{
Research on Transformation Characteristics of Binomial Coefficient Formula and Its Extended Model
}

\author{
Minghan Zhu' ${ }^{1}$, Jeffrey Zheng² \\ ${ }^{1}$ School of Software, Yunnan University, Kunming, China \\ ${ }^{2}$ Yunnan Laboratory of Quantum Information, Kunming, China \\ Email: crystalj000@163.com, conjugatelogic@yahoo.com
}

How to cite this paper: Zhu, M.H. and Zheng, J. (2019) Research on Transformation Characteristics of Binomial Coefficient Formula and Its Extended Model. Journal of Applied Mathematics and Physics, 7, 2927-2932.

https://doi.org/10.4236/jamp.2019.711202

Received: July 20, 2019

Accepted: November 26, 2019

Published: November 29, 2019

\begin{abstract}
Combinatorial methods are used to give specific mathematics, and the proof of combinatorial identities is the hot spot research of combinatorial mathematics. Binomial coefficients play an important role in the fields of physics, mathematics and computer science. In variances of rotation and reflection is the core characteristic for combinatorial systems. This paper uses different parameters to form reflection, rotation and specific features by using variant construction model and method, meanwhile the quantitative distribution characteristics of binomial coefficient formula are analyzed by three-dimensional maps. Using variant construction, the combinatorial clustering properties are investigated to apply binomial formulas and sample distributions, and various combinatorial patterns are illustrated. It is proved that the basic binomial coefficient formula and its extended model have obvious properties of reflection and rotation invariance.
\end{abstract}

\section{Keywords}

Binomial Coefficient, Spatial Extension, Variant Construction, Reflection, Rotation

\section{Introduction}

There are one-to-one relationships between binomial coefficients and PASCAL triangles. Mathematicians from ancient to modern are deeply interested with them from the angle of in variances [1]. Using visual technology of computer graphics and image processing, data streams are transformed into graphics or 
images as maps that could be displayed on the screen, and then various theory, method and technology are developed for interactive processing [2].

The resulting distribution with the family of that binomial formula and its extended model contain obvious invariant properties of reflection and rotation in the paper. It is expected that this kind of invariant formula can be closely associated with the invariant properties in quantum mechanics, classical physics, mathematics, computer science and convolutional neural network, which could be helpful to explore the optimization research of various applied computing methods [3].

\section{Theoretical Basis}

\subsection{Binomial Coefficient and Combinatorial Number}

Binomial coefficient [4]: or combinatorial numbers, is defined as, the $m$-th coefficient of expanded $x$ (Where $\mathrm{n}$ is a natural number and $\mathrm{m}$ is an integer).

Combinatorial number [5]: the number of all combinations of $m(m \leq n)$ elements selected from $\mathrm{n}$ different elements. It means the number of combinations of $m$ elements from $n$ different elements on the symbol expressed [6].

\subsection{Variant Construction}

The variant construction [7] proposed in 2010 is composed of three parts: variant logic, variant measurement and variant map. Variant logic is a new logic system based on 0 - 1 vector model.

Based on the traditional vector state, the system extends its phase spaces using two kinds of vector operations: permutation and complementarity. The $\mathrm{n}$ logical function space is extended from a traditional function space to a functional configuration space by two extended logic operations.

Variant measurements and variant maps have been applied to different problems, including classical cryptographic sequence analysis, quantum cryptographic sequence detection, chromosome whole gene sequence illustration, electrocardiogram signal detection, bat signal processing and other applications [7].

This paper focuses attention mainly to extend a basic binomial formula in variant measurement [7].

\subsection{Reflection and Rotation Invariants}

The reflection transformation [8] is one significant part of conversion in the Euclid plane geometry. The axial reflection on the plane and the mirror reflection are both called the reflection transformation [9].

Rotation invariant [10]: After the center coordinate is determined, the rotation operator rotates 360 degrees to coincide with the original image in the Euclid plane geometry. Meanwhile, the 90-degree rotations are most common in the discrete geometry applications. The rotation operation in this paper is limited to the 90 degree rotation and does not involve other rotation operations at any angle. 


\section{Process Flow}

\section{Derive from Binomial Coefficient Formula}

Ordered structure of four measures: $\{a, b, c, d\}$ in the monograph on variant constructions [7] pages 39-50. There are 192 different ways of representing structures under both permutation and combination. Any one of arbitrary structure corresponds to a set of binomial coefficients.

In this paper, the binomial formula $f(m, p, k)=\left(\begin{array}{c}m-p \\ k\end{array}\right)\left(\begin{array}{l}p \\ k\end{array}\right)$ is selected, and the value range is $0 \leq p \leq m ; 0 \leq k \leq p ; k \leq m-p$.

According to the binomial coefficient formula, multiple steps of transformations on different values contain various features of reflection and rotation invariances. Different binomial coefficient formulas are shown in four regions with distinguished characteristics. On a set of four binomial coefficient formula, their parameters and valued ranges are different. The transforming process for the four Zones \{I, II, III, IV $\}$ is shown in Figure 1.

The Zone I can be calculated from the formula. Range of Zone III can be carried out by reflecting binomial coefficients of corresponding positions in Figure 2. To simulate this type of reflection effects on the Zone III, the formula f3 can be applied.

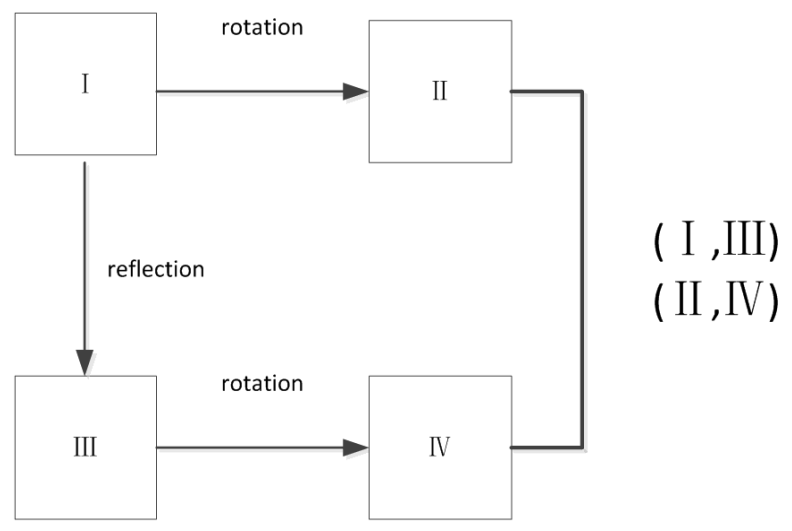

Figure 1. Process flow on a set of binomial coefficient formula.

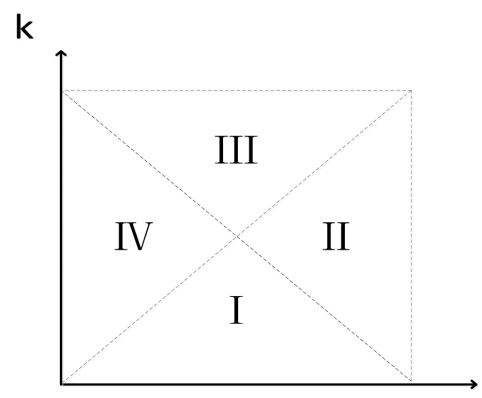

p

Figure 2. Conversion process of reflection and rotation from binomial and extended formulas. 
Because of reflection and rotation invariance between Zone I, III and Zone II, IV, rotated from Zone I, III to Zone II, IV. The binomial coefficients of the four regions are symmetric with the diagrams on $m-p=k$ and $p=k$.

In Figure 2, the four Zones and their relevant positions are systematically illustrated. Formula for each Zone and corresponding parameters are listed as follows.

1) $f(m, p, k)=\left(\begin{array}{c}m-p \\ k\end{array}\right)\left(\begin{array}{l}p \\ k\end{array}\right), 0<p<m ; 0<k<p ; k<m-p$;

2) $f_{3}(m, p, k)=\left(\begin{array}{c}m-k \\ p\end{array}\right)\left(\begin{array}{c}k \\ p\end{array}\right), 0 \leq k \leq m ; 0 \leq p \leq k, k \leq m-p$;

3) $f_{2}(m, p, k)=\left(\begin{array}{c}k \\ m-p\end{array}\right)\left(\begin{array}{c}m-k \\ m-p\end{array}\right), 0 \leq p \leq m ; 0 \leq k \leq p ; k>m-p$;

4) $f_{4}(m, p, k)=\left(\begin{array}{c}p \\ m-k\end{array}\right)\left(\begin{array}{c}m-p \\ m-k\end{array}\right), 0 \leq k \leq m ; 0 \leq p \leq k ; k>m-p$.

\section{Further Results}

In three-dimensional representation:

The distribution of different values is analyzed and discussed visually by using the measurement diagram in the combinatorial coefficients.

Applying the function $f_{1} m=11$, a total of 42 points are in the region of $12^{\star} 12$ shown in Figure 3(a) and Figure 3(b) and the distribution presents symmetric features. The color increases from blue to red. It can be clearly observed that there are two points at the highest level, the tangent values are equal, and there is no highest point in the top view Figure 3(a). Therefore, top view Figure 3(d) shows the distribution of the highest points.

When $\mathrm{m}=11$ and $\mathrm{m}=10$, the three-dimensional visualization results of function $\mathrm{f} 1$ are shown in Figures 3(a)-(d).

For Zone II, $\mathrm{m}=11$ and $\mathrm{m}=10$ as examples to compare the coefficient distribution by the rule of odd-even after reflection binomial formula.

Finally, for Zone II and IV, rotation effects are illustrated by the extended binomial coefficient distributions shown in Figures 4(a)-(d) through $f_{2}$ and $f_{4}$, as the rotation of $f_{1}$ and $f_{3}$ functions on rotation invariant.

There is no vertex at the top of the triangle on the top view, and the highest value is represented for two numerical points when $m$ is odd. The top view triangle has the highest vertex when $m$ is even.

\section{Conclusion}

It is a novel approach to explore rotation and reflection patterns using the four binomial coefficient formulas to show their coefficient distribution characteristics as reflection and rotation transformations. It is important to distinguish reflection and rotation characteristics from the selected coefficient binomial formulas and sample distributions following the condition of parameter transformations on 


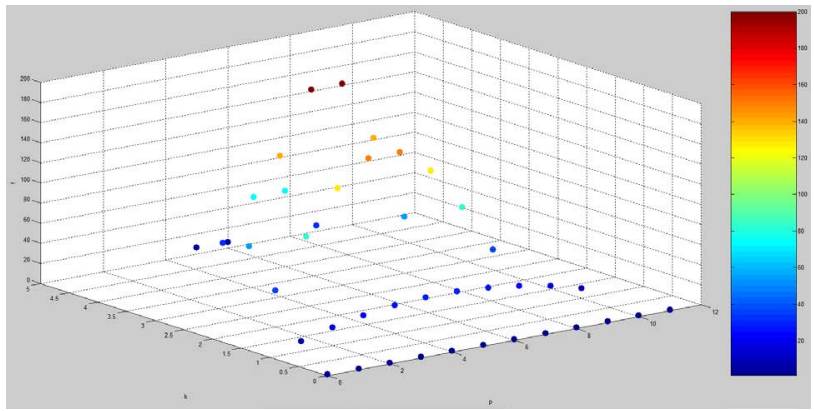

(a)

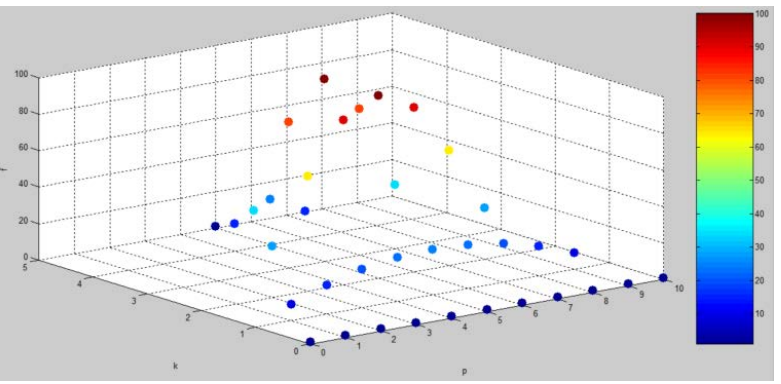

(c)

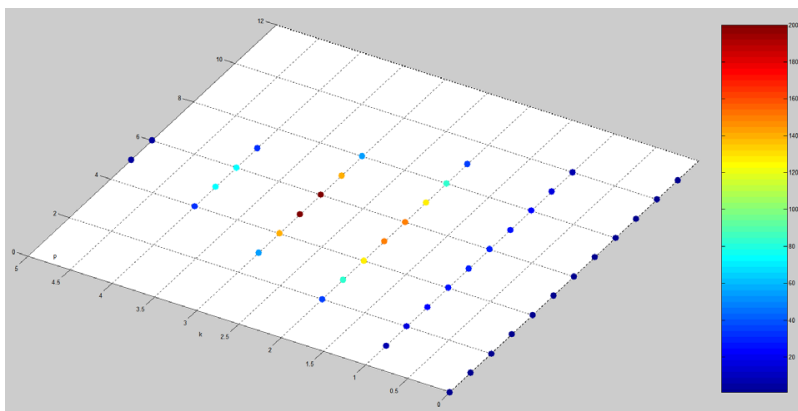

(b)

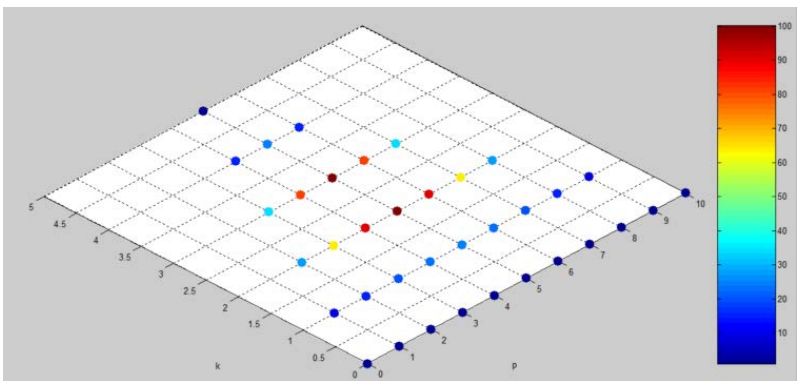

(d)

Figure 3. Three dimensional maps of initial binomial coefficient formula (a) $m=11$ in front view; (b) $m=11$ in top view; (c) $m=$ 10 in front view; (d) $m=10$ in top view.

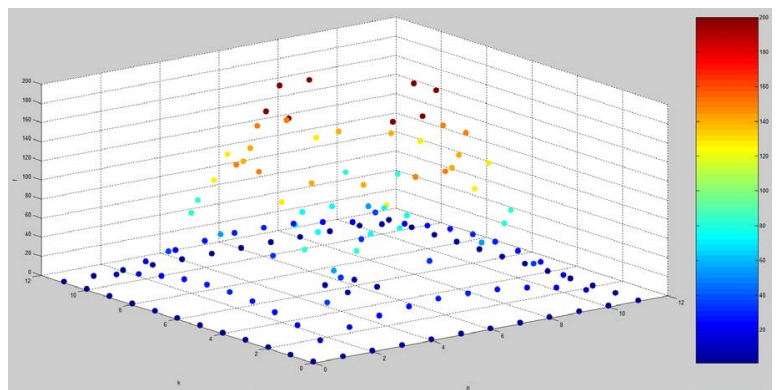

(a)

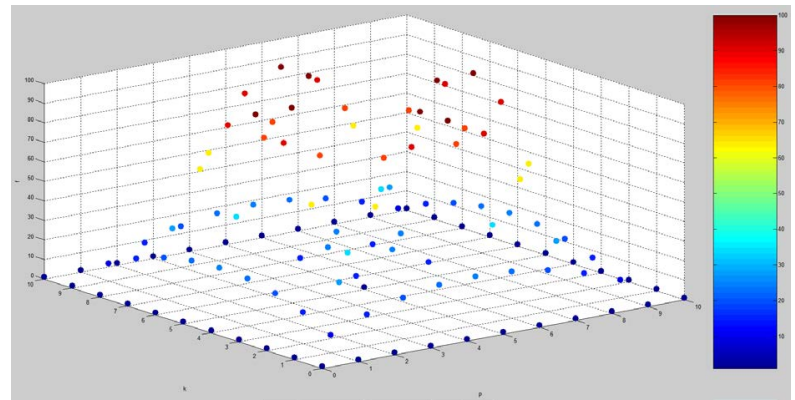

(c)

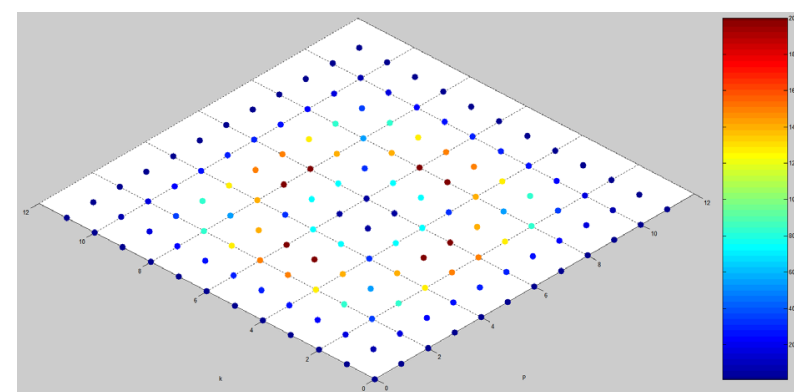

(b)

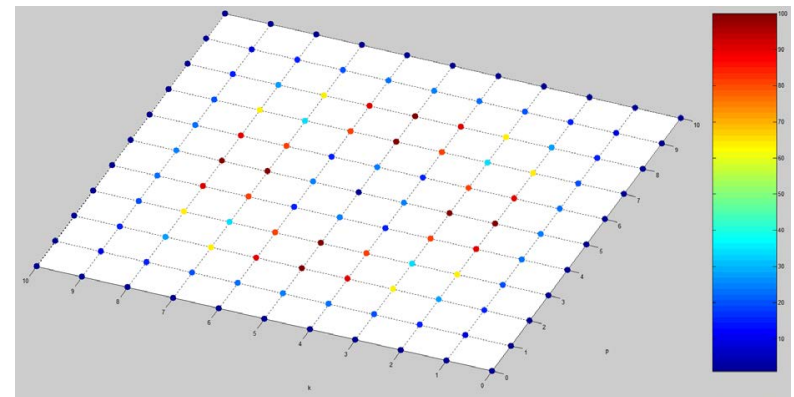

(d)

Figure 4. Three dimensional maps of the four Zones (a) $m=11$ in front view; (b) $m=11$ in top view; (c) $m=10$ in front view; (d) $m=10$ in top view.

odd and even numbers. A series of sample results are illustrated to explore the potential expansion ability of variant constructions to form a set of new binomial formula. It lays a theoretical foundation for the basic and applied research on 
big data analysis and simulation targets with complex combinatorial measurements in the future.

\section{Acknowledgements}

This project is supported by The Key Project of Quantum Communication Technology of Yunnan (2018ZI002) and Yunnan overseas high-level Scholar project.

\section{Conflicts of Interest}

The authors declare no conflicts of interest regarding the publication of this paper.

\section{References}

[1] Brualdi, R.A. (2005) Combinatorial Mathematics. 4th Edition, China Machine Press.

[2] Pang, X.M. (2009) Application of Combinatorial Transformation in Equations, Polynomials and Simple Graphs. Nankai University, Tianjin.

[3] Morgan, F. (2009) Geometric Measure Theory. World Publishing Corporation.

[4] Wu, J. (2012) Beauty of Mathematics. Posts and Telecom Press.

[5] Knuth, D.E. (2011) The Art of Computer Programming (Volume 4: Combinatorial Algorithms, Part 1). National Defence Industry Press.

[6] Knuth, D.E. (2002) The Art of Computer Programming (Volume 1, 3rd Edition). National Defence Industry Press.

[7] Zheng, J.Z.J. (2019) Variant Construction from Theoretical Foundations to Applications. Springer, China, 39-50, 237-245.

https://link.springer.com/book/10.1007/978-981-13-2282-2 https://doi.org/10.1007/978-981-13-2282-2_3

[8] Wang, Q.P. (2008) Reflection Transformation in Metrological Logic. Fuzzy Systems and Mathematics, 32.

[9] Yin, S.H. (2012) Calculation of Moment of Inertia of Rigid Body by Bernoulli Coefficient Table. Science Journal of Normal University, 32.

[10] Zhao, X.Q. (2000) Vandermonde Convolution Formula Unified Form and Corresponding Hypergeometric Transformation. Journal of Dalian University of Technology, 40. 